\title{
Avances sobre nutrición y fertilidad en ganado lechero: Revisión
}

\section{Advances on nutrition and fertility in dairy cattle: Review}

\author{
Pedro Meléndez ${ }^{a *}$, Julián Bartoloméb
}

\begin{abstract}
RESUMEN
En esta revisión se analizan los últimos antecedentes sobre la relación entre la nutrición y fertilidad en ganado lechero. Se establece la importancia de los niveles sanguíneos de glucosa en el postparto temprano y su relación con la fisiología de algunas hormonas tales como la insulina, IGF-I y somatotrofina. Se resalta la importancia de la inmunosupresión de la vaca durante el periparto y su relación con el exceso de tejido adiposo que establece un estado pro-inflamatorio característico asociado con la aparición de enfermedades metabólicas y reproductivas, que afectan el rendimiento productivo y la fertilidad del ganado lechero.
\end{abstract}

PALABRAS CLAVE: Nutrición, Fertilidad, Preparto, Postparto, Enfermedades.

\begin{abstract}
In this review, new advances on the relationship between nutrition and fertility in dairy cattle are presented. The importance of glucose levels in the early postpartum and its relationship to the physiology of some hormones such as insulin, IGF-I and somatotropin are established. The importance of immunosuppression around parturition and its relationship with the excess of adipose tissue that institutes a characteristic pro-inflammatory state is highlighted. I mmunosuppression is associated with the onset of metabolic and reproductive diseases affecting productivity and fertility of the dairy herd.
\end{abstract}

KEY WORDS: Nutrition, Fertility, Periparturient diseases, Postpartum, Prepartum.

Recibido el 13 de abril de 2016. Aceptado el 21 de junio de 2016.

a Department of Veterinary Medicine \& Surgery. University of Missouri, Estados Unidos.

b Facultad de Medicina Veterinaria. Universidad Nacional de la Pampa. Argentina.

*Autor de correspondencia: melendezp@missouri.edu 


\section{INTRODUCCIÓN}

Los pilares fundamentales de cualquier sistema de producción animal son la nutrición, reproducción, sanidad, bienestar animal, genética y recursos humanos. Ellos se interrelacionan íntimamente y determinan la eficiencia productiva y la rentabilidad del sistema. La relación entre la nutrición y la fertilidad ha sido ampliamente estudiada en todo el mundo y aun continua siendo un área de considerable investigación. Una adecuada fertilidad no se verá expresada si la nutrición y el manejo alimentario son sub-óptimos. En esta revisión se abordarán algunos aspectos básicos y generales que caracterizan esta relación en el ganado bovino lechero. Particular énfasis se indicará cuando haya diferencias marcadas entre hatos manejados en confinamiento o en base a pastoreo.

\section{Conceptos básicos}

La fertilidad es un concepto amplio y complejo y sus indicadores son muy variados y relativos de interpretar. El indicador más certero, que abarca a la mayoría de ellos, es la tasa de preñez (TP) cada 21 días, y refleja el riesgo de una vaca de preñarse en 21 días. Puede estimarse multiplicando la tasa de detección de celos por la tasa de concepción (TDC $x$ TC). Este indicador es ideal para sistemas continuos y con inseminación artificial (IA), y más difícil de interpretar en hatos que usan monta natural y que además concentran sus pariciones, típico de sistemas de pastoreo. Una nutrición óptima se verá reflejada en una mayor TP siempre y cuando el bienestar del animal y el manejo general sea el adecuado(1) $^{\text {. }}$

El manejo nutricional se debe enfocar hacia una óptima producción de leche, sin descuidar la sanidad y la fertilidad del animal. Cuando se alimenta solo para optimizar la producción de leche, la fertilidad se puede ver deteriorada. Esta relación inversa ha sido reportada en forma consistente ${ }^{(2,3)}$.

Debido al avanzado progreso genético, las vacas lecheras en la actualidad pueden incluso llegar a producir cantidades de leche inimaginables para el ser humano. En la raza Holstein, por ejemplo, se han reportado producciones de hasta más de $15,000 \mathrm{~kg}$ de leche por lactancia o $50 \mathrm{~kg}$ de leche/vaca/día en promedio, con producciones al pico de lactancia de
80 L/día. Desafortunadamente, la capacidad de consumo de alimento de esta vaca es limitada como para cubrir este nivel de producción y el animal debe recurrir a sus reservas corporales ${ }^{(4,5)}$. Al mismo tiempo, el animal debe priorizar aquellos procesos fisiológicos vitales. En este caso, los procesos reproductivos son secundarios para el animal cuando se trata de producir leche ${ }^{(6)}$. Los efectos de una nutrición inadecuada puede afectar la condición corporal (CC), aumentar la incidencia de anestro y disminuir las $\mathrm{TC}^{\text {(7-10). }}$.

Los sistemas de producción en base a pastoreo se establecen en regiones donde las condiciones ambientales permiten buen crecimiento de praderas, y el costo de oportunidad por el uso de la tierra es bajo. En este escenario, el manejo nutricional de las vacas a pastoreo se va a ver influenciado principalmente por la carga animal. El desafío es lograr la carga animal óptima que logre la mayor producción por vaca y por hectárea, sin afectar los procesos reproductivos, la salud y el bienestar de las vacas ${ }^{(11,12)}$. La evaluación de los sistemas productivos se debe basar más en los sólidos (kilogramos) que los litros de leche producidos (litros o kilogramos), ya que la adición de agua a la leche tiene un costo adicional. Por ejemplo, una vaca que produce $500 \mathrm{~kg}$ de grasa en un volumen de 5,000 $\mathrm{kg}$ de leche, va a ser más eficiente que una vaca que produce la misma cantidad de grasa en 7,500 kg de leche. Esto es debido a que la segunda vaca requiere sintetizar $120 \mathrm{~kg}$ adicionales de lactosa $(2,500 \mathrm{~kg}$ de leche $\times 48 \mathrm{~g}$ de lactosa) ${ }^{(12)}$. Debido a que la disponibilidad y el valor nutritivo de la pradera varía a través del año, el manejo reproductivo de la vaca lechera se deberá acomodar a las características de crecimiento y calidad de la pradera a través del año(13).

\section{Efecto de los nutrientes esenciales sobre la producción de leche y fertilidad}

Los aspectos nutricionales a considerar para optimizar la producción de leche y la fertilidad del ganado lechero son la energía, la fibra, la proteína, los minerales, las vitaminas y el agua de bebida.

La energía es un concepto abstracto, pero que se puede entender analizando las reservas corporales a través del depósito de tejido graso en 
zonas anatómicas estratégicas, tales como la base de la cola, la zona de la pelvis y los procesos transversales de las vértebras lumbares y costillas del animal. Estas reservas grasas se conocen como CC, y su estimación se ha utilizado como una herramienta simple que permite evaluar la nutrición energética del animal. La más utilizada es la escala de 1 a 5 con incrementos de $1 / 4$ de punto, siendo el valor 1 un animal emaciado y el valor 5 un animal extremadamente obeso(14). Durante el ciclo productivo la vaca lechera debe parir con una CC de 3.25 a 3.5 y no bajar a menos de 2.5 a los 60 a 90 días en leche. Si el animal pierde más de una unidad entre el parto y los 100 días en leche, verá comprometida su fertilidad $^{(15,16)}$. La pérdida en CC durante el postparto es un fenómeno normal, debido a que los requerimientos son mayores a los aportes ofrecidos por la dieta y al nivel de consumo de alimento del animal. Así, la vaca debe recurrir a sus reservas corporales para suplir la deficiencia dietaria y de consumo de alimentos. No obstante si la CC al parto es extremadamente baja el animal no podrá expresar su máximo potencial productivo, y si es excesivamente alta (obesidad) el animal tendrá problemas de distocia, menor consumo de alimento y mayor incidencia de enfermedades metabólicas tales como hígado graso y cetosis(13,17-21). Después del pico de producción, la leche empieza a disminuir en forma paulatina, y el animal es capaz de consumir mayores cantidades de alimento. Así, la vaca comienza a recuperar la CC que se perdió durante el postparto. Si la vaca se ha preñado se debe secar a los siete meses de gestación, para dar un descanso y preparación de dos meses a la glándula mamaria para la siguiente lactancia. El animal debería ser secado con una CC 3.0 a 3.25 y recuperar en 2 meses $1 / 4$ de CC necesaria para llegar con una condición al parto de 3.25 a 3.5. El animal nunca debe perder CC durante el periodo seco. En el caso de buscar recuperar CC durante el periodo seco en vacas que se secan muy delgadas $(C C \leq 2.75)$ se recomienda la aplicación de un bolo intraruminal de liberación lenta de monensina(1,22). Además, durante los últimos 21 días de gestación se debe empezar la adaptación del animal a las condiciones de producción y dietas altamente energéticas que recibirá después del parto ${ }^{(15,23,24)}$.
La proteína es un nutriente que debe ser considerado tanto en cantidad como en calidad. Una vaca requiere proteína tanto degradable como no degradable en el rumen. La proteína es esencial para la producción de leche, ya que el animal requiere de aminoácidos esenciales para la síntesis de caseína y otras proteínas menores de la leche ${ }^{(25)}$. Tanto la deficiencia como un exceso de proteína tienen un impacto negativo en la fertilidad del ganado bovino lechero. Una deficiencia va a afectar dramáticamente la producción de leche, pero también la fertilidad del animal. Un exceso de proteína también ha demostrado ser detrimental para la fertilidad de las vacas en producción ${ }^{(26,27)}$. Todo exceso de proteína es finalmente eliminado vía excremento o convertido en urea en el hígado del animal. La urea puede ser reciclada hacia el rumen del animal y reutilizada para la síntesis de proteína microbiana. No obstante, el exceso de urea podría ser tóxico para el ambiente uterino y oviducto, afectando tanto los gametos como el embrión, con la consiguiente reducción en fertilidad. Un exceso relativo de proteína también puede ocurrir cuando la dieta es deficiente en energía. Esto se va a traducir en mayores producciones de amonio ruminal y urea en el cuerpo del animal, produciendo el mismo efecto negativo mencionado ${ }^{(28)}$. El tópico sobre el exceso de urea y su impacto negativo en fertilidad ha sido muy controvertido. En algunos estudios se ha demostrado que valores de nitrógeno ureico en leche (46\% de la urea) mayores a 16 a $19 \mathrm{mg} / \mathrm{dl}$, sobre todo en los meses de verano, se han asociado con fertilidad reducida ${ }^{(26)}$; no obstante otros estudios no han encontrado esta asociación negativa ${ }^{(29)}$. Últimamente se ha dilucidado que la correlación fenotípica entre el nitrógeno ureico en leche y la fertilidad es casi cero, e incluso la correlación genotípica entre ambas características es levemente positiva, sugiriendo que niveles elevados de urea en leche se asocian a niveles elevados de fertilidad en el ganado lechero ${ }^{(30)}$. Por lo tanto, independientemente de la asociación entre urea y reproducción, la nutrición proteica debe ser balanceada en calidad y cantidad, incluyendo el balance aminoacídico, y debe ir acompañada de un aporte energético suficiente para optimizar la síntesis proteica microbiana a nivel ruminal(31). 
Los minerales son importantes componentes estructurales, de enzimas, y cofactores que permiten que el organismo funcione en forma adecuada, incluyendo los procesos reproductivos. Los minerales se requieren en cantidades absolutas y el principal obstáculo es conocer el porcentaje del mineral consumido que es absorbido y utilizado por parte del animal. En general los microminerales son más difíciles de balancear en condiciones prácticas de manejo nutricional y por ende se manejan en forma de premezclas, tanto añadidas a la dieta total como en comederos especiales donde el animal consume a voluntad(25). Los micro minerales con cierto rol directo en los procesos reproductivos son el $\mathrm{Zn}, \mathrm{Se}, \mathrm{Cu}, \mathrm{Co}$ y $\mathrm{Mn}$. En caso de deficiencias o excesos se han reportado casos de abortos, mortalidad embrionaria, anestro, y quistes ováricos ${ }^{(32,33)}$. Al igual que los minerales, las vitaminas son nutrientes esenciales que se requieren en cantidades pequeñas. Las vitaminas $A, D$ y $E$ deben ser aportadas en la dieta porque el animal no las sintetiza; en cambio las vitaminas del complejo B y la vitamina $C$ pueden ser producidas por los microorganismos del rumen, y por lo tanto, solo requieren ser aportadas en situaciones extremas de producción de leche ${ }^{(25)}$. Las vitaminas A y E son muy importantes para los procesos reproductivos (retención de membranas fetales, mortalidad embrionaria, repetición de calores, etc. ), sobre todo relacionado al estrés oxidativo que ocurre durante el periparto(23,34). En general las vitaminas son ofrecidas en forma de premezclas en conjunto con los minerales. También se pueden inyectar si esto es necesario ${ }^{(25)}$. Aunque la energía, fibra y proteína se encuentren balanceadas y aportadas adecuadamente, la respuesta productiva del animal, salud y fertilidad no serán óptimas si descuidamos los minerales y las vitaminas. De este punto se desprende que quizás el método que más se adecua a aportar una dieta balanceada en todos los nutrientes, son las dietas completas ofrecidas por un carro mezclador. Si la dieta se prepara debidamente y se ofrece en forma homogénea, el animal podrá consumir todos los nutrientes sin poder seleccionar a su gusto. Desafortunadamente, en sistemas pastoriles, los concentrados ofrecidos en la sala de ordeño o sobre los forrajes en comederos, no permiten un consumo homogéneo de todos los nutrientes, lo cual puede desfasar la fermentación ruminal y a ciertos disturbios digestivos, tales como acidosis ruminal, deficiencia en la síntesis de proteína microbiana, indigestiones simples, etc. ${ }^{(35)}$ Es por esto que el sistema ideal para animales a pastoreo, sería suplementación con una ración completa antes o después del ordeño, sobre todo en otoño e invierno, mínima cantidad de concentrado en sala y luego la pradera para complementar lo que falta.

A pesar de existir una asociación negativa entre producción de leche y fertilidad, se debe enfatizar que no es una relación de causa y efecto, y por ende hay situaciones en que hatos bien manejados pueden presentar niveles productivos extremadamente altos (42 a $44 \mathrm{~L}$ promedio por vaca al día) con TP sobre el $20 \%{ }^{(3,36)}$. En un estudio de California, EE.UU. ${ }^{(37)}$, se analizaron más de 6,400 vacas en producción y se observó que aquellas vacas en el cuartil inferior de producción ( $32 \mathrm{~kg} /$ día) presentaron una menor probabilidad de estar ciclando a los 65 días postparto que vacas que se encontraban en el cuartil 2 (39 kg/día), 3 (43,6 kg/día) y 4 (50 kg/día) de producción de leche, por lo tanto se concluyó que los factores de manejo pueden ser más limitantes para la fertilidad de vacas lecheras contemporáneas que su nivel genético per se.

Las vacas de alta producción son más susceptibles a desórdenes de tipo metabólico y enfermedades durante el periparto ${ }^{(1)}$. Estas enfermedades se asocian a mayores pérdidas de CC durante el postparto, y elevados niveles de ácidos grasos no esterificados (NEFA) al parto. Estas son vacas que presentan una marcada inmunosupresión, con mayores incidencias de retención de membranas fetales, metritis, mastitis, hipocalcemia e hipomagnesemia, cetosis, desplazamientos del abomaso e hígado graso. Además son animales que se tardan más en normalizar su ciclicidad postparto, con una TC reducida y por ende una tasa de preñez disminuida(3,13,16,28,38-42).

Aquellas vacas que logran adaptarse en forma exitosa desde su estado preparto al proceso de lactancia y que pueden evitar desbalances metabólicos y fisiológicos, van a ser capaces de sostener una alta producción de leche y lograr una fertilidad y salud adecuada. Por lo tanto aquellas vacas de alta producción y que presentan problemas 
de fertilidad, se puede atribuir a un balance energético negativo más severo, debido a un inadecuado manejo del periodo de transición o a un incremento en la incidencia de enfermedades del periparto $^{(21,43,44)}$.

En el caso de los sistemas a pastoreo, donde el objetivo es hacer coincidir requerimientos con aportes de la pradera, un $90 \%$ del rebaño debería parir en un periodo de 12 semanas a finales de invierno y principios de primavera.

Este es el típico sistema mono estacional neozelandés, que también se lleva a cabo en muchos países de Latinoamérica. Para lograr el objetivo planteado se debe lograr que: (i) todas las vacas hayan parido antes de iniciarse la época de servicio; (ii) $\geq 70 \%$ de las vacas estén ciclando al inicio del servicio; (iii) $\geq 90 \%$ de las vacas deberían entrar en un programa de IA a tiempo fijo (IATF) en los primeros 21 días del periodo de servicio; (iv) la TP para las primeras seis semanas debería ser $\geq 70 \%$ y para las 12 semanas de $\geq 90 \%$; (v) la duración del servicio $\leq 12$ semanas. Bajo este escenario, claramente las vacas deben recuperar prontamente su ciclicidad postparto y tener una alta probabilidad de establecer y mantener la preñez después de la monta o I $\mathrm{A}^{(12,15)}$.

\section{Signos generales de nutrición inadecuada}

Balance energético y condición corporal. El balance energético postparto es uno de los factores más significativos que influyen el estado reproductivo de los hatos lecheros. En general las vacas durante el postparto temprano pierden entre un 30 a $40 \%$ de su CC, pero en casos extremos pueden llegar a perder hasta un $80 \%$ de las reservas corporales bajo situaciones de un mal manejo nutricional ${ }^{(12,15)}$. En general, las vacas manejadas bajo pastoreo son más delgadas, ya que pierden más CC durante el postparto que vacas manejadas bajo confinamiento y alimentadas con raciones completas. Esta conclusión se corrobora con estudios llevados a cabo en la Universidad de Florida en hatos a pastoreo, donde la incidencia de vacas con $\geq 0.7 \mathrm{mM}$ de NEFA y $\geq 0.96 \mathrm{mM}$ de betahydroxi-butirato (BHB) durante el periodo postparto fue de 20 y $35.4 \%$ respectivamente, siendo que en hatos confinados los valores no sobrepasaron el 15 y $20 \%$, respectivamente ${ }^{(44,45)}$. En concordancia con estos resultados, en un estudio realizado en el sur de Chile ${ }^{(46)}$, las vacas que fueron sometidas a un pastoreo más intensivo y menor suplementación de concentrado, presentaron mayores concentraciones de NEFA y perdieron más CC que vacas que consumieron menos forraje y fueron suplementadas con más concentrado. Por otra parte, en un estudio llevado a cabo en I rlanda(11), en hatos con partos de parición de primavera, tanto la probabilidad de IA como de concepción se asociaron en forma positiva con el mínimo valor de CC (nadir) alcanzado durante el postparto, y la CC durante la época de montas o IA. Por lo tanto, una CC adecuada al parto (3.0 a 3.5) y una pérdida de CC postparto de menos de 0.5 puntos se asocian con un mejor rendimiento reproductivo y fertilidad en ganado lechero a pastoreo ${ }^{(12,17)}$.

\section{Efecto del estatus metabólico postparto sobre la reproducción}

El estado metabólico postparto, algunas hormonas y ciertos factores de crecimiento tienen un rol fundamental sobre los procesos reproductivos de la vaca lechera. Concentraciones en sangre reducidas de insulina, IGF-I, leptina y glucosa y concentraciones en sangre elevadas de BHB, NEFA y glucocorticoides se han asociado con un rendimiento reproductivo reducido y una mayor presencia de enfermedades en ganado lechero ${ }^{(15,39,43)}$. Sin embargo, se ha dilucidado que al seleccionar genéticamente por fertilidad, no hay una asociación directa con una mejora en el balance energético postparto de la vaca, a pesar de haberse encontrado diferencias en algunos metabolitos y hormonas, sugiriendo que el estado metabólico intrínseco dentro de la vaca es más importante que las diferencias observadas en su balance energético postparto(3,10,12). Así, se ha visto en ganado lechero que el IGF-I comienza a disminuir dos semanas antes del parto, y se acompaña con una disminución de la insulina e incrementos en los niveles de la hormona de crecimiento. Esta última, se asocia con una serie de cambios metabólicos en la vaca lechera, incluyendo una reducción en la sensibilidad de los tejidos a la insulina, incremento en la gluconeogénesis hepática y reducción del uso de glucosa en los tejidos, exceptuando la glándula mamaria ${ }^{(43,47)}$. 
Es bien sabido que el balance energético negativo en el postparto de la vaca lechera se asocia a una reducción en los pulsos de GnRH y LH, afectando el crecimiento, maduración y ovulación del folículo. Por otro lado, las vacas de alta producción tienen un mayor flujo sanguíneo y un metabolismo hepático más acelerado, que determina una desaparición más rápida de las hormonas reproductivas, afectando la calidad del ovocito y desarrollo del embrión. Además, la reducción en los niveles de estradiol deprimen la expresión del celo y los de progesterona (P4) se asocian a mayor mortalidad embrionaria ${ }^{(48-50)}$.

\section{El rol de la glucosa}

La glucosa ha tenido un especial interés en los últimos años como un metabolito clave en los procesos reproductivos de la vaca lechera. Una vaca lechera típica Holstein americana de alto mérito genético que produce $70 \mathrm{~kg}$ de leche al pico de lactancia, requiere de $5 \mathrm{~kg}$ de glucosa al día. Los rumiantes permanecen en un constante estado de gluconeogénesis a nivel hepático, ya que la mayoría de los carbohidratos son fermentados a ácidos grasos volátiles (AGV) en el rumen y el propionato es la base fundamental para la producción de glucosa a nivel del hígado. No obstante, los AGV solo aportan el $85 \%$ de la glucosa requerida y el resto debe provenir de algunos aminoácidos, lactato y glicerol, que está disponible cuando los NEFA son liberados a circulación. Además, la vaca cuenta con otros mecanismos tales como un estado temporal de insulino-resistencia, permitiendo solo a los tejidos que son independientes de insulina a utilizar la glucosa, como es la glándula mamaria. En este sentido, la vaca en postparto permanece en un estado de bajos niveles de glucosa, bajos niveles de insulina, IGF-I, y altos niveles de NEFA y

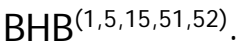

En ganado a pastoreo, los niveles de glucosa sanguínea también son menores en vacas de mayor producción que en vacas de menor producción ${ }^{(53)}$. La glucosa es fundamental para la producción y liberación de $\mathrm{GnRH}$ a nivel hipotalámico. Se ha demostrado que al inhibir la glicolisis a nivel neuronal, resulta en una menor frecuencia de los pulsos de LH producto de una inhibición en la síntesis de GnRH. Además, el efecto negativo de la hipoglicemia a nivel hipotalámico se ve potenciado por altos niveles de estrógenos, indicando que el efecto "feedback" (-) per se de los estrógenos sobre la liberación de LH se potencia con niveles bajos de glucosa sanguínea ${ }^{(43)}$. Durante la lactancia temprana, aquellas vacas con un elevado mérito genético para características de fertilidad, presentaron mayores concentraciones de glucosa circulante que vacas con menor mérito genético(10). Esta asociación se ha corroborado en un estudio, donde se reporta que las concentraciones de glucosa plasmática fueron mayores durante la primera semana postparto en aquellas vacas que se preñaron a la primera IA, comparado con aquéllas que fallaron al primer servicio ${ }^{(52)}$.

También se ha observado que la glucosa es crítica para una adecuada maduración del ovocito, afectando la expansión del cumulo, la maduración nuclear, la división celular y el subsecuente desarrollo del blastocito(54-56). Lo mismo se ha observado con un elevado nivel de NEFA sanguíneo que pueden afectar negativamente la fisiología del ovario, el folículo y el futuro embrión ${ }^{(43,57)}$.

El efecto beneficioso de la glucosa en el postparto temprano sobre la reproducción, solo se puede analizar como una asociación y no como mecanismo de causa y efecto. Esto se debe a que la glucosa se relaciona a muchos mecanismos metabólicos y hormonales que pueden enmascarar su efecto per se sobre la fertilidad. De hecho, los niveles de glucosa al momento de la primera IA ya no se asocian a las respuestas reproductivas de la $\operatorname{vaca}^{(43,52) \text {. }}$

\section{El rol de la insulina}

Las concentraciones sanguíneas de insulina en ganado lechero se encuentran reducidas en animales con balance energético negativo, tanto en vacas a pastoreo como confinadas y en vacas bajo estados de subnutrición ${ }^{(12)}$. En la medida que la lactancia progresa, los niveles de insulina incrementan y los de la hormona de crecimiento disminuyen ${ }^{(58)}$. El eje somatotrófico, que incluye a la hormona de crecimiento, sus receptores y el IGF-I se encuentra desacoplado en vacas de alta producción durante el postparto temprano, por lo tanto el hígado no responde a los efectos de la 
hormona de crecimiento y por ende se produce una menor cantidad de IGF-I. Este desacoplamiento se entiende en el contexto del proceso de la "partición de nutrientes" que permite una mayor producción de leche, afectando la reproducción en forma negativa $^{(43)}$. En el caso de hatos con parición continua durante el año, se puede tolerar cierta ineficiencia reproductiva, debido a que la vaca puede tener muchas más oportunidades de ser cubierta y obtener una preñez. Sin embargo, en hatos a pastoreo con parición estacional, una baja TC va a aumentar el número de animales eliminados que no se preñan durante la temporada de servicios establecida ${ }^{(47,59)}$.

La insulina también juega un rol fundamental en la esteroidogénesis ovárica, estimulando receptores tanto a nivel de células de la granulosa, como de los tejidos tecales y del estroma ovárico. Durante la lactancia temprana, también se ha visto que las vacas seleccionadas genéticamente por una alta fertilidad presentan mayores concentraciones de insulina en comparación a vacas con un mérito genético inferior. Consecuentemente, la insulina estimula la síntesis de IGF-I en vacas lecheras durante el inicio de la lactancia ${ }^{(12,43,58)}$.

\section{El rol del / GF-I}

El IGF-I es estructuralmente similar a la proinsulina y es un potente péptido/hormona proteica de tipo anabólica, la cual refleja el estado nutricional de un animal(58). El IGF-I afecta el crecimiento y maduración folicular, demostrando estar íntimamente asociado a la tasa de concepción tanto en animales en confinamiento como animales a pastoreo ${ }^{(2,60)}$. Vacas con concentraciones de IGF-I durante la primera semana postparto $>25 \mathrm{ng} / \mathrm{ml}$ y durante el primer servicio $>50 \mathrm{ng} / \mathrm{ml}$ tuvieron 11 y 5 veces más probabilidades de preñarse, respectivamente, que aquellas vacas con una menor concentración ${ }^{(61)}$. Vacas a pastoreo seleccionadas genéticamente por alta/baja fertilidad, presentaron mayores concentraciones de IGF-I. En este contexto, tanto la insulina como el IGF-I son indicadores metabólicos críticos para la fertilidad postparto de la vaca lechera. Ambas péptidos/hormonas proteicas se encuentran reducidas en vacas con balance energético negativo severo(43).

\section{El rol de la leptina}

Esta hormona proteica de 167 aminoácidos, producida esencialmente por los adipocitos, juega un rol fundamental en la modulación nutricional y metabólica de los tejidos. La leptina se ha llegado a asociar a mayores concentraciones séricas de gonadotrofinas. Durante el postparto temprano los niveles sanguíneos de leptina se encuentran reducidos debido al balance energético negativo y movilización grasa que ocurre en este periodo. No obstante el rol de la leptina en la regulación de los procesos reproductivos en ganado lechero aún no se ha dilucidado por completo(12).

\section{El rol de los NEFA, BHB y triglicéridos hepáticos}

La típica movilización grasa postparto se traduce en mayores concentraciones de NEFA. Los NEFA son captados por el hígado, los cuales son parcial o totalmente oxidados a nivel mitocondrial o re-esterificados en los hepatocitos a triglicéridos. Un exceso de NEFA a nivel hepático resulta en una acumulación neta de triglicéridos hepáticos, debido a la exportación disminuida de triglicéridos en la forma de lipoproteínas de muy baja densidad, clásica en bovinos. La oxidación parcial de los NEFA acompañada con bajos niveles de glucosa sanguínea, se traduce en una mayor síntesis de cuerpos cetónicos, principalmente BHB, debido a la falta de oxaloacetato a nivel de ciclo de Krebs. Tanto los niveles elevados en sangre de NEFA, como de BHB y la acumulación excesiva de triglicéridos en el hígado se asocia a alta/baja infertilidad ${ }^{(5,15)}$. Esta asociación negativa se puede deber a una mayor incidencia de enfermedades del periparto que experimentan las vacas con altos niveles de NEFA. Un estudio llevado a cabo en la zona central de Chile reportó que vacas con altos niveles de NEFA al parto tuvieron mayor incidencia de retención de membranas fetales y mastitis, y éstas a su vez están asociadas con infertilidad y mortalidad embrionaria en el ganado lechero ${ }^{(39,62)}$. De la misma forma, en un estudio llevado a cabo en el sur de Chile se observó que la suplementación con cebada en vacas a pastoreo resultó en menor concentración de BHB y mayor de glucosa durante el postparto, los cuales son factores determinantes para una buena 
fertilidad del ganado en el primer tercio de lactancia(63).

En el caso de vacas postparto a pastoreo que presentaron valores de NEFA $\geq 0.7 \mathrm{mM}$ tuvieron una menor probabilidad de estar ciclando a los 50 días postparto y de preñarse a la primera IA posparto(64). Lo mismo se ha reportado en hatos manejados bajo estabulación total(45,65). Así, se observó que por cada $0.1 \mathrm{mM}$ de aumento en el BHB sanguíneo a la semana 1 y 3 postparto, la proporción de vacas preñadas se redujo en un 2 y $3 \%$, respectivamente ${ }^{(20)}$. Por otro lado, también se reportó que la TP de un ciclo de 21 días se redujo en un $0.9 \%$ en aquellos hatos que presentaron más de un $15 \%$ de las vacas muestreadas con valores de NEFA $\geq 0.7 \mathrm{mM}$ durante el periodo posparto(45).

Tanto los NEFA como el BHB inducen a inmunosupresión a través de la reducción en la fagocitosis y la capacidad oxidativa de los neutrófilos. También se ha observado que estos dos metabolitos se asocian a un retardo de la involución uterina y mayor incidencia de metritis, relacionado a una alteración de la expresión de genes claves en la recuperación del endometrio ${ }^{(18,41,66)}$.

\section{El rol del sistema inmune}

El sistema inmune de la vaca en transición se deprime en forma inherente al parto, sobre todo cuando se alcanza un máximo nivel de estrés, que es el proceso de parto del animal. Sin embargo, bajos ciertas situaciones, esta depresión puede ser mucho más severa y por ende puede significar el desarrollo de enfermedades infecciosas típicas del periparto, tales como la mastitis, metritis y neumonías ${ }^{(23,67)}$.

Los factores de tipo metabólico que se han asociado a una mayor depresión del sistema inmune de la vaca periparto son el exceso de las concentraciones plasmáticas de NEFA y cuerpos cetónicos durante el postparto. J unto con aquello, se sabe hoy en día que el tejido adiposo además de almacenar energía tiene un rol mecánico, regenerativo, endocrino e inmunológico bien determinado ${ }^{(67,68)}$. El tejido adiposo bajo la epidermis y sobre todo el que rodea a órganos vitales, tales como el riñón o grasa intra-abdominal mesentérica y omental, juega un rol inmunológico, y mecánico protectivo fundamental. Una vez estimulado, el adipocito libera NEFA y citoquinas proinflamatorias. Así, la obesidad y el envejecimiento se asocian con activación inmunitaria del tejido graso y liberación de citoquinas, tales como el factor de necrosis tumoral, la interleuquina 1 y 6 , alteración de la sensibilidad a la insulina y lipolisis. Este estado se conoce como inflamación sistémica "esteril" (no infeccioso), con desregulación metabólica y lipotoxicidad. Vacas obesas al parto presentarán no solo mayor incidencia de problemas metabólicos, sino también problemas de tipo inflamatorioinfeccioso ${ }^{(68,69)}$.

\section{El rol del calcio y la salud uterina postparto}

El rol del calcio ionizado sobre la musculatura lisa y estriada es fundamental para un adecuado proceso de transición ${ }^{(70)}$. En un estudio estadounidense se observó que un $25 \%$ de las vacas primíparas y más de un $50 \%$ de las vacas multíparas, experimentan un estado de hipocalcemia subclínica (Ca total $<8.0 \mathrm{mg} / \mathrm{dl}$ ) durante las primeras $48 \mathrm{~h}$ posparto ${ }^{(71)}$. Estos niveles deprimidos de calcio afectan la contractibilidad del miometrio y la actividad fagocitaria y bactericida de los neutrófilos, con un mayor riesgo de desarrollarse retención de membranas fetales y metritis puerperal ${ }^{(38,70,72)}$.

\section{Mecanismos que ligan el metabolismo con los procesos reproductivos}

Son dos los procesos fundamentales que se deben reestablecer adecuadamente durante el postparto, para lograr una fertilidad satisfactoria y que se asocian íntimamente a los mecanismos metabólicos de la vaca: el restablecimiento de la ciclicidad posparto y la involución del útero $(15,41,43)$.

Existe una asociación directa entre los niveles de insulina y del IGF-I con el momento del inicio de la ciclicidad posparto. En el ovario, tanto la insulina como el IGF-I promueven la proliferación, diferenciación y sobrevida de las células foliculares, y a su vez estas hormonas se asocian directamente con los niveles sanguíneos de glucosa. Además, estos metabolitos se relacionan con un adecuado 
nivel de gonadotrofinas a nivel hipofisario y GnRH a nivel hipotalámico, las cuales ejercen su efecto positivo sobre las estructuras del ovario(43,58). Por otro lado, el estatus metabólico y hormonal del posparto temprano se asocia a una inmunosupresión típica que afecta los procesos involutivos del útero. En un posparto normal, el útero se debe contraer en forma adecuada y la mucosa uterina se debe infiltrar adecuadamente de tejido linfoide, para establecer una limpieza de los tejidos placentarios y patógenos presentes en el útero. Sin embargo, las células inmunes son dependientes de los niveles sanguíneos de glucosa; y niveles sanguíneos excesivos de NEFA y BHB afectan tanto los procesos de fagocitosis $y$ quimiotaxis, como la capacidad oxidativa para destruir bacterias ${ }^{(43,67)}$. También se ha observado que los neutrófilos en el posparto contienen menos glicógeno y menor actividad celular inmune ${ }^{(73)}$.

Ya establecida la fecundación del oocito, el embrión debe proceder a su implantación, y la vaca debe llevar adelante el reconocimiento temprano de la preñez, que ocurre alrededor del día 17 postfecundación. Es bien sabido que los niveles sanguíneos de P4 durante la primera semana post concepción se asocian positivamente con un establecimiento exitoso de la preñez. La P4 es fundamental para la actividad del histótrofo a nivel de mucosa uterina. Si este tejido glandular no se desarrolla en forma adecuada, debido a bajos niveles de P4, el embrión se desarrolla en forma más lenta, lo que resulta en una menor producción de interferón tau, compuesto esencial para un adecuado reconocimiento de la presencia del embrión en el útero. En vacas de alta producción, con una mayor filtración hepática de hormonas esteroidales, se presentan niveles más bajos de P4 durante el periodo posparto ${ }^{(50,56,74)}$.

Por otro lado, se ha visto que los embriones y fetos de vacas lactantes contienen menos niveles de glucosa que aquellos fetos de la misma edad gestacional pero de vacas no lactantes. Si los niveles de glucosa se observan reducidos en vacas preñadas y lactantes, es lógico pensar que el estado de preñez se verá afectado si los niveles de glucosa son menores a los normales ${ }^{(43)}$.

\section{CONCLUSIONES}

El éxito reproductivo depende de la coordinación de una serie de eventos fisiológicos, que incluyen la restauración del útero, reanudación de la ciclicidad postparto, desarrollo de un folículo y ovocito viable, ovulación, fertilización, y adecuado desarrollo embrionario y fetal para llegar a un proceso normal de parto. Dentro de estos complejos mecanismos, los niveles sanguíneos de insulina, IGF-I, glucosa, NEFA y BHB posparto son fundamentales. Por otro lado, un adecuado nivel de hormonas reproductivas $(\mathrm{GnRH}, \mathrm{LH}, \mathrm{FSH}$, estrógenos y progesterona) son imprescindibles dentro de estos mecanismos reproductivos.

\section{LITERATURA CITADA}

1. Melendez P, Risco CA. Reproduction, events and management pregnancy: Periparturient disorders. Reference module in food sciences. First ed. Elsevier Academic Press; 2016.

2. Lucy MC. Loss in high-producing dairy cattle: Where will it end? J Dairy Sci 2001;84:1277-1293.

3. Berry DP, Friggens NC, Lucy M, Roche JR. Milk production and fertility in cattle. Annu Rev Anim Biosci 2016; 15:269-290.

4. Drackley JK. Biology of dairy cows during the transition period: The final frontier? J Dairy Sci 1999;82:2259-2273.

5. Drackley JK, Overton TR, Douglas GN. Adaptations of glucose and long-chain fatty acid metabolism in liver of dairy cows during the periparturient period. J Dairy Sci 2001;84(E. Suppl):E100-E112.

6. Bell AW. Regulation of organic nutrient metabolism during transition from late pregnancy to early lactation. J Anim Sci 1995; 73:2804-2819.

7. Castro N, Kawashima C, van Dorland HA, Morel I, Miyamoto A, Bruckmaier RM. Metabolic and energy status during the dry period is crucial for the resumption of ovarian activity postpartum in dairy cows. J Dairy Sci 2012;95:5804-5812.

8. Kawashima C, Matsui M, Shimizu T, Kida K, Miyamoto A. Nutritional factors that regulate ovulation of the dominant follicle during the first follicular wave postpartum in high-producing dairy cows. J Reprod Develop 2012;58: 10-16.

9. Cardoso FC, LeBlanc SJ, Murphy MR, Drackley JK. Prepartum nutritional strategy affects reproductive performance in dairy cows. J Dairy Sci 2013;96:5859-5871.

10. Moore SG, Fair T, Lonergan P, Butler ST. Genetic merit for fertility traits in Holstein cows: IV. Transition period, uterine health and resumption of cyclicity. J Dairy Sci 2014; 97:2740-2752.

11. Buckley F, O'Sullivan K, Mee JF, Evans RD, Dillon P. Relationships among milk yield, body condition, cow weight, and reproduction in spring calved Holstein-Friesians. J Dairy Sci 2003;86:2308-2319.

12. Butler ST. Nutritional management to optimize fertility of dairy cows in pasture-based systems. Animal 2014;8,s1:15-26. 
13. Roche JR, Meier S, Heiser A, Mitchell MD, Walker CG, Crookenden MA, et al. Effects of precalving body condition score and prepartum feeding level on production, reproduction, and health parameters in pasture-based transition dairy cows. J Dairy Sci 2015;98:71647182.

14. Ferguson JD, Galligan DT, Thomsen N. Principal descriptors of body condition score in Holstein cows. J Dairy Sci 1994; 77:2695-2703.

15. Drackley JK, Cardoso FC. Prepartum and postpartum nutritional management to optimize fertility in high-yielding dairy cows in confined TMR systems. Animal 2014;8,s1:5-14.

16. Akbar H, Grala TM, Vailati Riboni M, Cardoso FC, Verkerk G, McGowan J , et al. Body condition score at calving affects systemic and hepatic transcriptome indicators of inflammation and nutrient metabolism in grazing dairy cows. J Dairy Sci 2015; 98:1019-1032.

17. Roche JR, Macdonald KA, Burke CR, Lee J M, Berry DP. Associations among body condition score, body weight, and reproductive performance in seasonal-calving dairy cattle. J Dairy Sci 2007; 90:376-391.

18. Wathes DC, Fenwick M, Cheng Z, Bourne N, Llewellyn S, Morris DG, et al. Influence of negative energy balance on cyclicity and fertility in the high producing dairy cow. Theriogenology 2007;68:S232s241.

19. Peter AT, Vos PL, Ambrose DJ . Postpartum anoestrus in dairy cattle. Theriogenology 2009; 71:1333-1342.

20. Walsh SW, Williams EJ, Evans AC. A review of the causes of poor fertility in high milk producing dairy cows. Anim Reprod Sci 2011;123:127-138.

21. Wathes DC. Mechanisms linking metabolic status and disease with reproductive outcome in the dairy cow. Reprod Domest Anim 2012; 47:s4 304-312

22. Melendez P, Goff JP, Risco CA, Archbald LF, Littell R, Donovan GA. Pre-partum monensin supplementation improves body reserves at calving and milk yield in Holstein cows dried-off with low body condition score. Res Vet Sci 2007;82:349-357.

23. Goff JP. Major advances in our understanding of nutritional influences on bovine health. J Dairy Sci 2006;89:1292-1301.

24. Dubuc J, Duffield TF, Leslie KE, Walton JS, LeBlanc SJ . Risk factors and effects of postpartum anovulation in dairy cows. J Dairy Sci 2012; 95: 1845-1854.

25. National Research Council. Nutrient requirements of dairy cattle, $7^{\text {th }}$ revised ed. Washington, DC: National Academy Press; 2001.

26. Melendez $P$, Donovan $A$, Hernandez J. Milk urea nitrogen and infertility in Florida Holstein cows. J Dairy Sci 2000;83:459-463.

27. Sinclair KD, Garnsworthy PC, Mann GE, Sinclair LA. Reducing dietary protein in dairy cow diets: implications for nitrogen utilization, milk production, welfare and fertility. Animal 2014; 8:262-274.

28. Melendez P, Donovan A, Hernandez J, Bartolome J, Risco C, Staples C, Thatcher W. Milk, plasma, and blood urea nitrogen concentrations, dietary protein, and fertility in dairy cattle. J Am Vet Med Assoc 2003;223:628-634

29. Cheng Z, Oguejiofor CF, Swangchan-Uthai T, Carr S, Wathes DC. Relationships between circulating urea concentrations and endometrial function in postpartum dairy cows. Animals 2015;5:748-773.
30. Mucha S, Strandberg E. Genetic analysis of milk urea nitrogen and relationships with yield and fertility across lactation. J Dairy Sci 2011; 94:5665-5672.

31. Patton RA, Hristov AN, Lapierre $\mathrm{H}$. Protein feeding and balancing for amino acids in lactating dairy cattle. Vet Clin North Am Food Anim Pract 2014;30:599-621.

32. Wilde D. Influence of macro and micro minerals in the periparturient period on fertility in dairy cattle. Anim Reprod Sci 2006; 96:240-249.

33. Swecker WS J r. Trace mineral feeding and assessment. Vet Cl North Am Food Anim Prac 2014; 30:671-688.

34. Abuelo A, Hernández J, Benedito J L, Castillo C. The importance of the oxidative status of dairy cattle in the periparturient period: revisiting antioxidant supplementation. J Anim Physiol Anim Nutr (Berl) 2015;99:1003-1016.

35. Oelberg TJ, Stone W. Monitoring total mixed rations and feed delivery systems. Vet Clin North Am Food Anim Prac 2014;30:721744.

36. Pinedo P, Melendez P, Paudyal S, Arias F, Krauss R, Lopez H, Luco A, Vergara, C. Association between disease occurrence and fertility of dairy cows in three geographic regions of Chile. Theriogenology 2016; 86:817-823.

37. Santos JE, Rutigliano HM, Sá Filho MF. Risk factors for resumption of postpartum estrous cycles and embryonic survival in lactating dairy cows. Anim Reprod Sci 2009; 110:207-221.

38. Meléndez $P$, Donovan GA, Risco CA, Goff JP. Plasma mineral and energy metabolite concentrations in dairy cows fed an anionic prepartum diet that did or did not have retained fetal membranes after parturition. Am J Vet Res 2004;65: 1071-1076.

39. Melendez P, Marin MP, Robles J, Rios C, Duchens M, Archbald L. Relationship between serum nonesterified fatty acids at calving and the incidence of periparturient diseases in Holstein dairy cows. Theriogenology 2009; 72:826-833.

40. Esposito G, Irons PC, Webb EC, Chapwanya A. Interactions between negative energy balance, metabolic diseases, uterine health and immune response in transition dairy cows. Anim Reprod Sci 2014; 144:60-71.

41. LeBlanc SJ . Reproductive tract inflammatory disease in postpartum dairy cows. Animal 2014;8,s1:54-63.

42. Somers JR, Huxley J, Lorenz I, Doherty ML, O'Grady L. The effect of lameness before and during the breeding season on fertility in 10 pasture-based I rish dairy herds. Ir Vet J 2015;68:14-20.

43. Lucy MC, Butler ST, Garverick HA. Endocrine and metabolic mechanisms linking postpartum glucose with early embryonic and foetal development in dairy cows. Animal 2014;8,s1:82-90.

44. Ribeiro ES, Lima FS, Greco LF, Bisinotto RS, Monteiro APA, Favoreto $M$, et al. Prevalence of periparturient diseases and effects on fertility of seasonally calving grazing dairy cows supplemented with concentrates. J Dairy Sci 2013;96:5682-5697.

45. Ospina PA, Nydam DV, Stokol T, Overton TR. Association between the proportion of sampled transition cows with increased nonesterified fatty acids and beta-hydroxybutyrate and disease incidence, pregnancy rate, and milk production at the herd level. J Dairy Sci 2010;93:3595-3601.

46. Schöbitz J, Ruiz-Albarrán M, Balocchi OA, Wittwer F, Noro M, Pulido RG. Effect of increasing pasture allowance and concentrate 
supplementation on animal performance and microbial protein synthesis in dairy cows. Arch Med Vet 2013;45:247-258.

47. Lucy MC, Verkerk GA, Whyte BE, Macdonald KA, Burton L, Cursons RT, Roche JR, Holmes CW. Somatotropic axis components and nutrient partitioning in genetically diverse dairy cows managed under different feed allowances in a pasture system. J Dairy Sci 2009; 92:526-539.

48. Wiltbank MC, Souza AH, Carvalho PD, Bender RW, Nascimento AB. Improving fertility to timed artificial insemination by manipulation of circulating progesterone concentrations in lactating dairy cattle. Reprod Fert Develop 2011;24:238-243.

49. Wiltbank MC, Souza AH, Carvalho PD, Cunha AP, Giordano JO, Fricke PM, et al. Physiological and practical effects of progesterone on reproduction in dairy cattle. Animal 2014;8,s1:70-81.

50. Crowe MA, Diskin MG, Williams EJ. Parturition to resumption of ovarian cyclicity: comparative aspects of beef and dairy cows. Animal 2014;8,s1:40-53.

51. Melendez $\mathrm{P}$, Risco CA. Management of transition cows to optimize reproductive efficiency in dairy herds. Vet $\mathrm{Cl}$ North Am Food Anim Prac 2005;21:485-501.

52. Garverick HA, Harris MN, Vogel-Bluel R, Sampson JD, Bader J, Lamberson WR, et al. Concentrations of nonesterified fatty acids and glucose in blood of periparturient dairy cows are indicative of pregnancy success at first insemination. J Dairy Sci 2013;96:181188.

53. Snijders SE, Dillon PG, O'Farrell KJ, Diskin MG, Wylie AR, O'Callaghan D, et al. Genetic merit for milk production and reproductive success in dairy cows. Anim Reprod Sci 2001;65:1731.

54. Leroy J L, Rizos D, Sturmey R, Bossaert P, Gutierrez-Adan A, Van Hoeck V, et al. Intrafollicular conditions as a major link between maternal metabolism and oocyte quality: a focus on dairy cow fertility. Reprod Fert Develop 2011;24:1-12.

55. Leroy J L, Vanholder T, Van Knegsel AT, Garcia-Ispierto I, Bols PE. Nutrient prioritization in dairy cows early postpartum: mismatch between metabolism and fertility? Reprod Domest Anim 2008; 43,s2:96-103.

56. Lonergan $\mathrm{P}$, Forde $\mathrm{N}$. Maternal-embryo interaction leading up to the initiation of implantation of pregnancy in cattle. Animal 2014; 8,s1:64-69.

57. Van Hoeck V, Sturmey RG, Bermejo-Alvarez P, Rizos D, GutierrezAdan $\mathrm{A}$, Leese $\mathrm{HJ}$, et al. Elevated non-esterified fatty acid concentrations during bovine oocyte maturation compromise early embryo physiology. PLoS One 2011;6:e23183.

58. Lucy MC. Functional differences in the growth hormone and insulinlike growth factor axis in cattle and pigs: implications for postpartum nutrition and reproduction. Reprod Domest Anim 2008; 43:31-39.

59. Cabrera VE. Economics of fertility in high-yielding dairy cows on confined TMR systems. Animal 2014;8:211-221.
60. Patton J, Kenny DA, McNamara S, Mee JF, O'Mara FP, Diskin MG, Murphy JJ. Relationships among milk production, energy balance, plasma analytes, and reproduction in Holstein-Friesian cows. J Dairy Sci 2007; 90:649-658.

61. Taylor VJ, Cheng Z, Pushpakumara PG, Beever DE, Wathes DC. Relationships between the plasma concentrations of insulin-like growth factor-I in dairy cows and their fertility and milk yield. Vet Rec 2004; 155:583-588.

62. Pinedo PJ, Melendez P, Villagomez-Cortes JA, Risco CA. Effect of high somatic cell counts on reproductive performance of Chilean dairy cattle. J Dairy Sci 2009;92: 1575-1580.

63. Pulido RG, Berndt S, Orellana P, Wittwer F. Effect of source of carbohydrate in concentrate on the performance of high producing dairy cows during spring grazing. Arch Med Vet 2007;39:19-26.

64. Ribeiro ES, Cerri RL, Bisinotto RS, Lima FS, Silvestre FT, Greco LF, et al. Reproductive performance of grazing dairy cows following presynchronization and resynchronization protocols. J Dairy Sci 2011;94:4984-4996.

65. Chapinal N, Leblanc SJ, Carson ME, Leslie KE, Godden S, Capel M, Santos JE, Overton MW, Duffield TF. Herd-level association of serum metabolites in the transition period with disease, milk production, and early lactation reproductive performance. J Dairy Sci 2012; 95:5676-5682.

66. Ster C, Loiselle MC, Lacasse P. Effect of postcalving serum nonesterified fatty acids concentration on the functionality of bovine immune cells. J Dairy Sci 2012; 95: 708-717.

67. Sordillo LM. Nutritional strategies to optimize dairy cattle immunity J Dairy Sci 2016;99:1-16.

68. Tchkonia T, Thomou T, Zhu Y, Karagiannides I, Pothoulakis C, J ensen MD, Kirkland J L. Mechanisms and metabolic implications of regional differences among fat depots. Cell Metab 2013;17:644656.

69. Sordillo LM, Raphael W. Significance of metabolic stress, lipid mobilization, and inflammation on transition cow disorders. Vet Clin North Am Food Anim Pract 2013;29:267-278.

70. Goff JP. Calcium and magnesium disorders. Vet Clin North Am Food Anim Pract 2014;30:359-381.

71. Reinhardt TA, Lippolis JD, McCluskey BJ, Goff JP, Horst RL. Prevalence of subclinical hypocalcemia in dairy herds. Vet J 2011; 188:122-124.

72. Kimura K, Reinhardt TA, Goff JP. Parturition and hypocalcemia blunts calcium signals in immune cells of dairy cattle. J Dairy Sci 2006; 89:2588-2595.

73. Galvão KN, Flaminio MJ, Brittin SB, Sper R, Fraga M, Caixeta L, et al. Association between uterine disease and indicators of neutrophil and systemic energy status in lactating Holstein cows. J Dairy Sci 2010; 93:2926-2937.

74. Lonergan $\mathrm{P}$. Influence of progesterone on oocyte quality and embryo development in cows. Theriogenology 2011;76:1594 1601. 